\title{
Structure-Function Relationships between Aldolase C/Zebrin II Expression and Complex Spike Synchrony in the Cerebellum
}

\author{
Shinichiro Tsutsumi, ${ }_{1}^{1}$ Maya Yamazaki, ${ }^{2}$ Taisuke Miyazaki, ${ }^{3}$ Masahiko Watanabe, ${ }^{3}$ Kenji Sakimura, ${ }^{2}$ Masanobu Kano, ${ }^{1}$ \\ and Kazuo Kitamura ${ }^{1,4}$ \\ ${ }^{1}$ Department of Neurophysiology, Graduate School of Medicine, The University of Tokyo, Tokyo 113-0033, Japan, ${ }^{2}$ Department of Cellular Neurobiology, \\ Brain Research Institute, Niigata University, Niigata 951-8585, Japan, ${ }^{3}$ Department of Anatomy, Hokkaido University Graduate School of Medicine, \\ Sapporo 060-8638, Japan, and ${ }^{4}$ PRESTO, JST, Saitama 332-0012, Japan
}

\begin{abstract}
Simple and regular anatomical structure is a hallmark of the cerebellar cortex. Parasagittally arrayed alternate expression of aldolase $\mathrm{C} /$ zebrin II in Purkinje cells (PCs) has been extensively studied, but surprisingly little is known about its functional significance. Here we found a precise structure-function relationship between aldolase $\mathrm{C}$ expression and synchrony of $\mathrm{PC}$ complex spike activities that reflect climbing fiber inputs to PCs. We performed two-photon calcium imaging in transgenic mice in which aldolase $\mathrm{C}$ compartments can be visualized in vivo, and identified highly synchronous complex spike activities among aldolase C-positive or aldolase C-negative PCs, but not across these populations. The boundary of aldolase $\mathrm{C}$ compartments corresponded to that of complex spike synchrony at single-cell resolution. Sensory stimulation evoked aldolase C compartment-specific complex spike responses and synchrony. This result further revealed the structure-function segregation. In awake animals, complex spike synchrony both within and between PC populations across the aldolase $\mathrm{C}$ boundary were enhanced in response to sensory stimuli, in a way that two functionally distinct $\mathrm{PC}$ ensembles are coactivated. These results suggest that $\mathrm{PC}$ populations characterized by aldolase $\mathrm{C}$ expression precisely represent distinct functional units of the cerebellar cortex, and these functional units can cooperate to process sensory information in awake animals.
\end{abstract}

Key words: climbing fiber; microzone; Purkinje cell; zone

\section{Introduction}

The cerebellar cortex has an elaborate parasagittal organization (Groenewegen et al., 1979; Voogd and Glickstein, 1998), and neighboring Purkinje cells (PCs) located within a certain parasagittal area tend to show synchronous complex spike activities (Bell and Kawasaki, 1972; Sasaki et al., 1989). This synchronous structure is thought to correspond to a cerebellar "microzone,"

Received May 26, 2014; revised 0ct. 20, 2014; accepted Nov. 23, 2014

Author contributions: S.T., M.K., and K.K. designed research; S.T., T.M., and M.W. performed research; M.Y. and K.S. contributed unpublished reagents/analytic tools; S.T. analyzed data; S.T., M.Y., T.M., M.W., M.K., and K.K. wrote the paper.

This work was supported by Grants-in-Aid for Scientific Research (23115504, 25560432, 25115705, and 25290003 to K.K. and 21220006 and 25000015 to M.K.), the Strategic Research Program for Brain Sciences (Development of Biomarker (andidates for Social Behavior), Comprehensive Brain Science Network, the Global COE Program (Integrative Life Science Based on the Study of Biosignaling Mechanisms) from MEXT Japan, and the Uehara Memorial Foundation (to K.K.). We thank M. Hashizume for technical assistance; M. Tada for participation in preliminary experiments; T. Ishikawa for providing analysis software (TaroTools for Igor Pro); T. Kawashima for advice on MATLAB routines; and Simon Schultz, Masanori Matsuzaki, Yoshikazu Isomuram, and members of the Kano lab for discussions.

The authors declare no competing financial interests.

K. Kitamura's present address: Department of Physiology, Graduate School Department of Interdisciplinary Research, University of Yamanashi, Chuo, Yamanashi 409-3898, Japan.

Correspondence should be addressed to either Kazuo Kitamura or Masanobu Kano, Department of Neurophysiology, Graduate School of Medicine, The University of Tokyo, 7-3-1 Hongo, Bunkyo-ku, Tokyo 113-0033, Japan, E-mail: kitamurak@yamanashi.ac.jp or mkano-tky@m.u-tokyo.ac.jp.

DOI:10.1523/JNEUROSCI.2170-14.2015

Copyright $@ 2015$ the authors $\quad 0270-6474 / 15 / 350843-10 \$ 15.00 / 0$ originally defined based on differential patterns of sensory responses (Andersson and Oscarsson, 1978). Synchronous complex spike activities are thought to arise from anatomical organization of olivo-cerebellar climbing fibers (Sugihara et al., 2001; Voogd et al., 2003) and electrical coupling between neurons in the inferior olive (Blenkinsop and Lang, 2006). Recent imaging studies with high spatial resolution have revealed spatially fine parasagittal structures with high complex spike synchrony (Mukamel et al., 2009; Ozden et al., 2009; Schultz et al., 2009; Ghosh et al., 2011) that correspond to microzones defined with classical electrophysiological techniques (Bell and Kawasaki, 1972; Andersson and Oscarsson, 1978). These imaging studies have shown that microzones are activated spontaneously or by sensorimotor inputs, indicating that microzones represent functional units in the cerebellar cortex. However, the precise relationship between microzones and anatomical structure of the cerebellar cortex is still unknown.

PCs heterogeneously express a variety of proteins and biological molecules to form longitudinal stripes within the cerebellum (Brochu et al., 1990; Oberdick et al., 1998; Hashimoto and Mikoshiba, 2003; Sarna et al., 2006; Sugihara and Quy, 2007). Among these molecules, aldolase $\mathrm{C} /$ zebrin II is most commonly used as a marker for these stripes. Seven aldolase C-positive $(1+$ to $7+)$ and -negative $(1-$ to $7-)$ compartments are known to exist in each hemicerebellum (Brochu et al., 1990). PCs in each aldolase 
C compartment receive projections from neurons located in specific subnuclei of the inferior olive (Sugihara and Shinoda, 2004). PCs in each aldolase $\mathrm{C}$ compartment in turn project to a distinct subdivision of the deep cerebellar nuclei (Sugihara, 2011), which sends inhibitory projections back to the inferior olive (de Zeeuw et al., 1989). Therefore each aldolase C compartment represents an anatomically connected olivo-cerebello-nuclear module. However, the functional significance of aldolase $\mathrm{C}$ compartmentalization still remains largely unexplored. A multi-electrode study has shown some correlation between complex spike synchrony and aldolase $\mathrm{C}$ compartments to a resolution of several hundred micrometers (Sugihara et al., 2007). However, the width of an aldolase C compartment can be up to $300-400 \mu \mathrm{m}$ in mice (Sugihara and Quy, 2007), and that of microzones may be as low as $100 \mu \mathrm{m}$ (Ozden et al., 2009). Studies with high spatial resolution are therefore essential to clarify the precise relationship between microzones and aldolase $\mathrm{C}$ compartments.

To elucidate the relationship between aldolase C compartments and complex spike synchrony at single-cell resolution, we visualized aldolase $\mathrm{C}$ compartments in vivo using novel transgenic mouse (Aldoc-tdTomato) and performed two-photon calcium imaging. We found a cell-level consistency between them, and thus concluded that aldolase $\mathrm{C}$ compartments precisely correspond to cerebellar zones. We also found that functionally distinct microzones could cooperate across zones during awake sensory processing.

\section{Materials and Methods}

Animals. All experiments were performed according to national guidelines and were approved by the Animal Experiment Committees of The University of Tokyo, Niigata University, and Hokkaido University. The Aldoc-tdTomato transgenic mice were produced by homologous recombination in RENKA C57BL/6N-derived ES cells (Mishina and Sakimura, 2007). We designed the targeting vector with a tdTomato protein sequence followed by a neomycin resistance cassette (Neo) inserted in frame just behind the translational initiation site of the aldolase $\mathrm{C}$ gene (Aldoc; Fig. 1A). This targeting vector was constructed with a $12 \mathrm{~kb}$ C57BL/6 genomic fragment $(6.4 \mathrm{~kb}$ upstream and $5.57 \mathrm{~kb}$ downstream from the translational initiation site) and diphtheria toxin gene (DT) using a BAC subcloning kit (Gene Bridges). ES cell clones with the correct recombination were identified by Southern blotting using the $5^{\prime}$-probe on EcoR V-digested genomic DNA and the 3'-probe on SacI-digested genomic DNA. Chimeric mice were generated as described previously (Fukaya et al., 2006). Heterozygous mice (Aldoc ${ }^{\text {tdTomato/+ }}$ ) were used for further analysis. No overt phenotype was detected in heterozygous mice, as described previously (Fujita et al., 2014). This mouse line is available upon request (contact to K.S. at sakimura@bri.niigata-u.ac.jp).

Immunohistochemistry. Under deep pentobarbital anesthesia, AldoctdTomato mice were transcardially perfused with $4 \%$ paraformaldehyde in $0.1 \mathrm{M}$ sodium phosphate buffer. Coronal microslicer sections were then processed (50 $\mu \mathrm{m}$ in thickness; VT1000S; Leica). For immunofluorescence, the sections were successively incubated in a free-floating state at room temperature with $10 \%$ normal donkey serum for $20 \mathrm{~min}$, goat calbindin antibody (a marker of PCs) and a rabbit anti-aldolase C antibody, and Alexa 488- and Cy5-conjugated species-specific secondary antibodies (1:200; Invitrogen; Jackson ImmunoResearch) for $2 \mathrm{~h}$. Images were taken with a confocal laser scanning microscope (FV1000; Olympus) equipped with a helium-neon/argon laser and a PlanApo $(10 \times /$ $0.40)$ and a PlanApoN $(60 \times / 1.42$, oil-immersion $)$ objective lens.

Animal preparation and in vivo two-photon imaging. Adult heterozygous Aldoc-tdTomato mice $(>\mathrm{P} 60)$ of both sexes were anesthetized with ketamine/xylazine $(100 / 10 \mathrm{mg} / \mathrm{kg})$. We chose this anesthesia for calcium imaging because of the ease in experimental preparation (e.g., easier to control bleeding and less brain swelling compared with isoflurane anesthesia). Animals were put on a warm blanket and body temperature was kept at $37^{\circ} \mathrm{C}$. The depth of anesthesia was constantly monitored by ob- serving forelimb pinch withdrawal reflexes, and additional injections of anesthetics were given as needed. The skin and muscles above the cerebellum were removed and a custom-made metal plate was fixed with Superglue and dental acrylic cement. An $\sim 2 \mathrm{~mm}$ craniotomy was performed over the left or right folium Crus IIa (4 mm lateral and $2 \mathrm{~mm}$ posterior to the occipital bone line) and the dura mater was carefully removed. Agarose (1.5\%) and a glass coverslip were mounted over the craniotomy to reduce motion artifacts due to respiration and heartbeat.

All the imaging experiments were performed using a custom-built two-photon microscope (Sutter Instruments) with $40 \times$ objective lens (Olympus) controlled by ScanImage software (Pologruto et al., 2003) for MATLAB (The MathWorks). Two-photon excitation was achieved with a pulsed Ti:sapphire laser [wavelength of $830 \mathrm{~nm}$ for Oregon Green 488 BAPTA-1 AM (OGB-1 AM) calcium imaging, $800 \mathrm{~nm}$ for Cal-520 calcium imaging, $950 \mathrm{~nm}$ for RFP imaging, $80 \mathrm{MHz}$ repetition rate, and 100 fs pulse width; Mai Tai, Spectra-Physics]. Average laser power was adjusted to be $<20 \mathrm{~mW}$ (typically $5-10 \mathrm{~mW}$ for calcium imaging $7-12 \mathrm{~mW}$ for tdTomato imaging) to avoid phototoxicity. Fluorescence signals were divided into green and red channels with a dichroic mirror and emission filters (Chroma), and detected with a pair of photomultiplier tubes (Hamamatsu)

We first imaged tdTomato fluorescence to identify the boundary of aldolase C compartments. Compartmental expression of tdTomato in PCs was easily visualized by two-photon microscopy (Fig. 1B). The characteristic width and sequence of mouse aldolase C compartments in Crus II (Sugihara and Quy, 2007) aided the identification and naming of each compartment. The entire location of all the compartments in the imaging field was manually mapped in advance because we later injected a red fluorescent dye, Alexa 594, which would blur the boundaries. A highresolution $3 \mathrm{D}$ stack ( $512 \times 512$ pixels, $220 \mu \mathrm{m}$ each, and $3 \mu \mathrm{m}$ per slice) was acquired from the pial surface to the PC soma on the aldolase $\mathrm{C}$ compartment boundary. This image stack was used as a reference for calcium imaging.

An AM ester calcium indicator dye (OGB-1 AM; Invitrogen or Cal520 AM, AAT BioQuest) was bulk loaded using a protocol as described previously (Hashizume et al., 2013; Tada et al., 2014). Briefly, OGB-1 AM or Cal-520 AM $(\sim 200 \mu \mathrm{M})$ was dissolved in DMSO with $10 \% \mathrm{w} / \mathrm{v}$ Pluronic F-127 (Invitrogen). The aliquot of dye was mixed with Alexa 594 fluorescent dye $(20 \mu \mathrm{M}$; Invitrogen) and placed into a glass pipette (4 $M \Omega$ ). The dye solution was injected into the cerebellar molecular layer. Two injections were performed at $15-20$ psi for 5 min using a Picospritzer (General Valve). Successful dye ejection was monitored through the Alexa channel on two-photon imaging. One to three hours after dye loading, calcium imaging was performed on the aldolase $\mathrm{C}$ compartment boundaries. The locations of the boundaries were roughly identified by using an aldolase $\mathrm{C}$ map obtained before dye injection. The pattern of blood vessels was compared with that present in the tdTomato images to precisely adjust the boundary locations. To obtain calcium transients from the PC dendrites, horizontal full-frame scan movies were acquired at $128 \times 128$ pixels resolution, and 1000 frames at $7.8 \mathrm{~Hz}$. In a subset of the calcium-imaging experiments, timed air-puff stimuli (40-45 psi for $50 \mathrm{~ms}$, every $10 \mathrm{~s}, 13$ times in $128 \mathrm{~s}$ ) were applied at the ipsilateral perioral surface using a Picospritzer.

Pharmacology. In a subset of calcium-imaging experiments, carbenoxolone (120 mg/kg; Sigma) or harmaline ( $1 \mathrm{mg} / \mathrm{kg}$; Sigma) was intraperitoneally injected during anesthesia. After baseline calcium imaging was performed, either of the two drugs was administered and then imaging was performed $30 \mathrm{~min}$ after the drug injection.

Imaging in awake mice. To perform imaging in awake mice, they were acclimated to the head fixation for $\sim 30$ min before imaging. After imaging under anesthesia, mice were allowed to recover from anesthesia and then imaging was performed during the awake state. Vigilance state was determined by monitoring sporadic active movements, e.g., whisking, grooming, eye blink, or limb and body movements. We determined that mice were awake if they were actively whisking and exhibited reflex body movements in response to the air puff stimuli.

Electrophysiology. An adult heterozygous Aldoc-tdTomato mouse (>P60) was anesthetized with isoflurane (1.0-1.5\%). A shadowpatching technique (Kitamura et al., 2008) was used for in vivo cell-attached re- 
cordings from PCs. A glass electrode filled with extracellular solution containing Alexa dye $(5 \mathrm{M} \Omega$ ) was navigated through the two-photon microscope and targeted at the PC soma. Approximately 5 min after the establishment of a loose seal, a current-clamp recording was performed for 2 min during simultaneous dendritic calcium imaging. Data were obtained using a Multiclamp 700B amplifier (Molecular Devices), filtered at $10 \mathrm{kHz}$, and digitized at $20 \mathrm{kHz}$ using a Digidata 1322A (Axon Instruments) controlled by AxoGraph X software (AxoGraph Scientific).

Data analysis. For the analysis of calcium imaging data, regions of interest (ROIs) corresponding to the active PC dendrites were detected using custom-made MATLAB routines (MATLAB R2013a; The MathWorks). We totally automated a previously described correlation-based detection method (Ozden et al., 2008; Schultz et al., 2009; Smith and Häusser, 2010) and succeeded in minimizing the time required for human supervision (typically 1-2 min for each dataset). For all the analyses, a correlation coefficient was calculated using the following equation:

$$
C_{i j(\tau)}=\frac{\sum_{t=0}^{T} X_{i(t)} X_{j(t+\tau)}}{\sqrt{\sum_{t=0}^{T}\left\{X_{i(t)}\right\}^{2} \sum_{t=0}^{T}\left\{X_{j(t)}\right\}^{2}}}
$$

where $X_{i}$ and $X_{j}$ are fluorescent traces of $i$ th and $j$ th pixels or dendrites, $T$ is total recording time, and $\tau$ is lag time between the two traces. $C_{i j}(0)$, cross-correlation coefficient at zero lag time, was defined as synchrony.

To extract the fluorescence changes corresponding to the calcium transients, the raw movie was first converted to $\Delta F / F$ wave, expressed as $\Delta F / F=\left(F-F_{0}\right) /\left(F_{0}-F_{b}\right)$, where $F_{0}$ is the baseline fluorescence in the absence of calcium transients and $F_{b}$ is the background fluorescence. $F_{0}$ was defined as follows. First, we calculated a threshold as mean +1 SD of the $F$ wave of each pixel, and then the $F$ wave below the threshold was averaged to obtain $F_{0}$. The minimum fluorescence of the stack was assigned to $F_{b}$, which usually corresponded to the pixel located within a blood vessel. The $\Delta F / F$ wave of each pixel was then high-pass filtered at $0.1 \mathrm{~Hz}$ to eliminate slow drifts presumably due to photo-bleaching effects or glial signals.

As PC dendrites elongate almost parallel to each other in a sagittal plane, we calculated the direction of the dendrites to aid autodetection of ROIs. The imaging field was segmented into sets of 4 pixel width strips, $0-45$ degrees from the vertical line. Correlation coefficients between the mean $\Delta F / F$ wave of each strip and that of each pixel within the strip were calculated and summed. The values calculated in certain sets of strips were further summed. The angle of the strip set with a maximal correlation value was the detected PC dendritic angle (Fig. $2 B b$ ).

The imaging field was again segmented into 6 pixel width $(10.3 \mu \mathrm{m})$ strips in the direction of the calculated angle. A summed correlation coefficient between the $\Delta F / F$ waves from all the pixels within a strip was plotted (Fig. 2Bc). Seed pixels (3-30) with the highest correlation value were recorded, and the mean correlation coefficient between the $\Delta F / F$ wave of seed pixels and that of all the pixels was plotted on each pixel. This procedure highlighted the shape of PC dendrites (Fig. $2 B d$ ). Images were then filtered and binarized using an adaptive threshold filter (adaptive threshold, available on-line at MATLAB central; window size: 256, constant: 0.1 ). ROIs were selected from the binarized image (Fig. $2 \mathrm{Be}$ ). Averaged $\Delta F / F$ waves within detected ROIs were calculated and apparent false positives (e.g., no calcium signals at all) were manually omitted to yield the final set of ROIs (Fig. $2 B f$ ).

For experiments with sensory stimulation, we defined responsive dendrites at a given trial as those satisfying the following two criteria. (1) Calcium transient peaks were observed between 0 and $128 \mathrm{~ms}$ after the onset of the air puff. This is because it takes $128 \mathrm{~ms}$ to acquire one frame and true complex spike responses should be $<128 \mathrm{~ms}$ in duration. (2) The peak exceeds the mean $+2 \mathrm{SD}$ from the baseline, which was calculated from $\Delta F / F$ waves $0-1 \mathrm{~s}$ before the onset of the air puff. Response probability for one dendrite was calculated from one imaging session (13 trials). The response probabilities of all the dendrites from all the mice were averaged in reference to the aldolase $\mathrm{C}$ boundaries (Fig. 5D). The synchrony of sensory-evoked responses was calculated using $\Delta F / F$ waves $0-2 \mathrm{~s}$ after onset of the air puff (Fig. $5 E$, top row). Spontaneous synchrony was calculated for the same time period in the absence of air puff stimulation (Fig. 5E, middle row).

Electrophysiological data analysis was performed using Igor Pro software (WaveMetrics). All statistical analyses were performed using R version 3.0.0 (http://www.r-project.org/).

\section{Results}

\section{Visualization of aldolase $\mathrm{C}$ compartments in vivo}

To visualize aldolase C-expressing PCs in intact mice, we used a targeted knock-in approach to generate the Aldoc-tdTomato mouse line. In the cerebellum of these mice, the red fluorescent protein, tdTomato, was expressed under the control of the aldolase C gene (Aldoc) promoter (Fig. 1A). Recently, a similar strategy was used to visualize aldolase $C$ expression using Aldoc-Venus knock-in mice (Fujita et al., 2014). Two-photon imaging of the cerebellar cortex in Aldoc-tdTomato mice revealed that the aldolase $\mathrm{C}$ compartment boundaries across the molecular layer to the PC layer were clear at single-cell resolution (Fig. 1B). To check whether the expression of tdTomato correctly reflected that of aldolase $\mathrm{C}$, we immunolabeled cerebellar sections of Aldoc-tdTomato mice with anti-aldolase $\mathrm{C}$ antibody. The results show that tdTomato is specifically and exclusively expressed in cells expressing aldolase $\mathrm{C}$ in the cerebellar cortex of Aldoc-tdTomato mice (Fig. 1C).

\section{Climbing fiber responses in the PC population in relation to aldolase $\mathrm{C}$ compartments}

To investigate the relationship between aldolase $\mathrm{C}$ compartmentalization and complex spike synchrony, PCs in the cerebellar folium Crus IIa of Aldoc-tdTomato mice were bulk loaded with OGB-1 or Cal-520. We chose Crus IIa because it is easy to access and has unique and consistent aldolase $\mathrm{C}$ banding with relatively wide compartments (4+ to 7+; Sugihara and Quy, 2007), which facilitates the analysis of synchrony between PC populations. Spontaneous dendritic calcium transients of PCs, which reflect complex spikes (Kitamura and Häusser, 2011; Fig. 2A), were recorded at boundaries of aldolase $\mathrm{C}$ compartments under anesthesia (Fig. 2C,D). We found a significant correlation between the synchrony of calcium transients and aldolase $\mathrm{C}$ compartments. Dendritic calcium transients were highly synchronized within aldolase C-positive PCs and within aldolase C-negative PCs (Fig. $2 D)$. The calcium transients were far less synchronized between the pairs across aldolase C-positive and -negative compartments. A color-coded correlation matrix clearly shows that the boundary of high-synchrony areas precisely corresponded to that of the aldolase $\mathrm{C}$ compartments, determined at single-cell resolution (Fig. 2E).

We next examined the location dependence of the relationship between boundaries of aldolase C compartment and those of synchrony of calcium transients. The synchrony was evaluated around the boundaries of aldolase $\mathrm{C}$ compartments from $5 \mathrm{a}+$ to $7+$ in Crus IIa. We found that boundaries of high-synchrony areas apparently corresponded to those of the aldolase $C$ compartments for $7+/ 6-, 5-/ 5+$, and $5+/ 5 a-$ (Fig. $3 A$ ). In contrast, boundaries of calcium transient synchrony for $6-/ 6+$ and $6+/ 5-$ were blurred and did not clearly correspond to those of the aldolase $\mathrm{C}$ compartments. These patterns were highly consistent across animals and across corresponding boundary of left and right lobules ( $5+/ 5-$ boundary; Fig. $3 A, D ; n=6$ mice each, respectively). Mean synchrony between PC dendrite pairs located $<100 \mu \mathrm{m}$ 
A

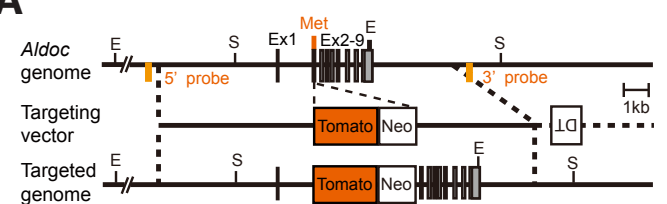

B

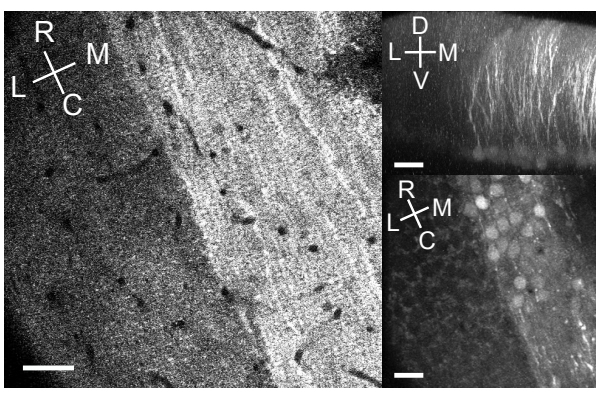

C
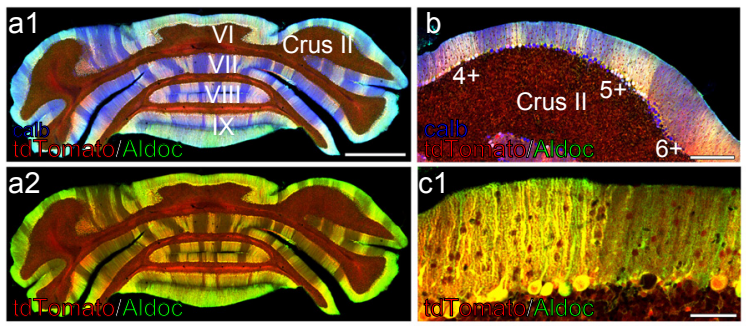

c2

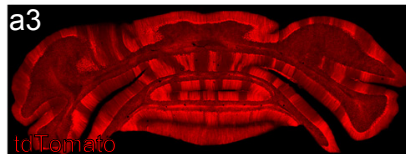

c2
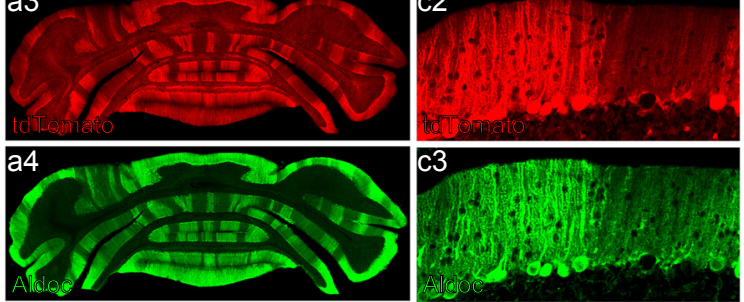

Figure 1. Aldolase C compartments are visualized in vivo. $A$, Knock-in construct of Aldoc-tdTomato mouse line. Gray boxes indicate exon 1-9. Met, initial methionine; Tomato, tdTomato coding sequence followed by a polyA addition signal sequence; Neo, pgk-gb2 neomycin resistance cassette; E, EcoR V; S, Sacl. B, Two-photon imaging of aldolase C compartments in vivo. A single horizontal plane in the molecular layer (left) and XZ (right top) and XY (right bottom) projection of the image stack. R, rostral; C, caudal; L, lateral; M, medial; D, dorsal; V, ventral. Scale bars: $40 \mu \mathrm{m}$. C, A representative coronal section of the cerebellum taken from an Aldoc-tdTomato mouse immunostained with anti-calbindin (blue) and anti-aldolase C (green) antibodies. Red fluorescence of tdTomato was overlaid. Scale bars: $\mathbf{C a}, 1 \mathrm{~mm} ; \boldsymbol{C b}, 200 \mu \mathrm{m} ; \boldsymbol{C c}, 50 \mu \mathrm{m}$.

A<smiles>CC=CC(C)(C)C</smiles><smiles>CC#[As]</smiles>

B

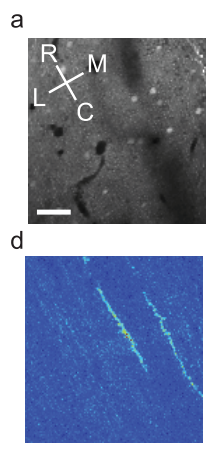

D

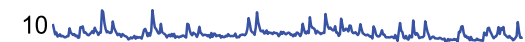

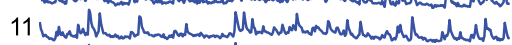

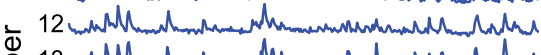

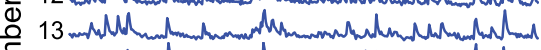

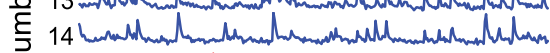

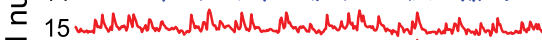

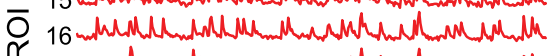

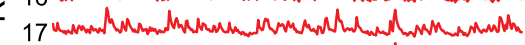

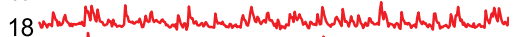

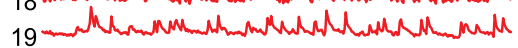

$$
\begin{aligned}
& \underset{10 \mathrm{sec}}{\mathrm{J}} 0.2 \Delta \mathrm{F} / \mathrm{F}
\end{aligned}
$$
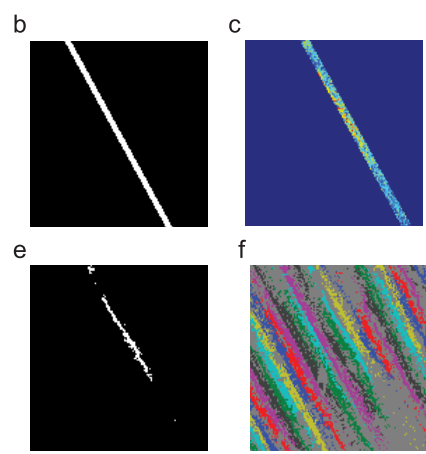

E

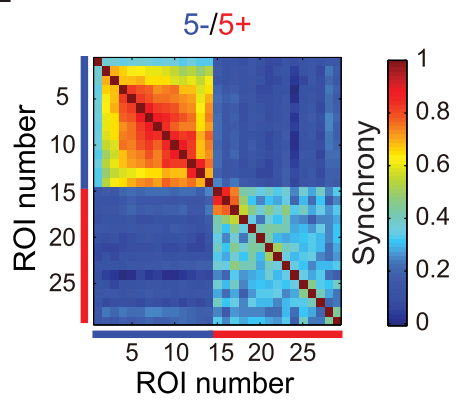

Figure 2. Aldolase ( compartment boundaries correspond to those determined by complex spike synchrony at single-cell resolution. $A$, Left, Example waveforms of simple spike and complex spike. Right, Simultaneous cell-attached recording (top) and dendritic calcium imaging (bottom) from the same PC. Blue asterisks represent complex spike peaks. SS, simple spike; CS, complex spike. $\boldsymbol{B} \boldsymbol{a}$, An example image from the raw movie of OGB-1 AM calcium imaging. $\boldsymbol{B} \boldsymbol{b}$, Detected angle of $\mathrm{PC}$ dendrites. $\boldsymbol{B} \boldsymbol{c}$, Color-coded correlation coefficient between a certain pixel and all the pixels in the strip. $\boldsymbol{B} \boldsymbol{d}$, Color-coded correlation coefficient between seed pixels and all the pixels in the imaging field. $\boldsymbol{B}$, Adaptive threshold filtered map from $\boldsymbol{B d}$. Only one ROI around the seed pixels was selected. $\boldsymbol{B} \boldsymbol{f}$, Pseudocolored map of the ROls. C, Calcium imaging was performed at a representative aldolase $C$ compartment boundary $(5-/ 5+)$. Pseudocolored ROIs (PC dendrites) are overlaid to the corresponding RFP image. $D$, Representative $\Delta F / F$ waves taken from $C$. Red and blue traces are from aldolase $C$-positive and -negative $\mathrm{PCS}$, respectively. $\boldsymbol{E}$, Correlation matrix calculated by using $\Delta F / F$ waves from all the dendrite pairs in $C$. Red and blue bars denote aldolase $C$-positive and -negative $P C S$, respectively. $R$, rostral; C, caudal; L, lateral; $M$, medial. Scale bars: $40 \mu \mathrm{m}$. 
A

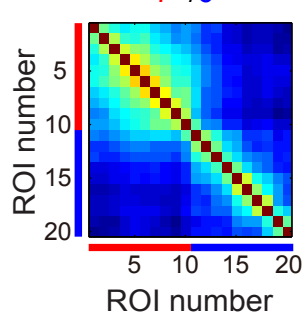

B

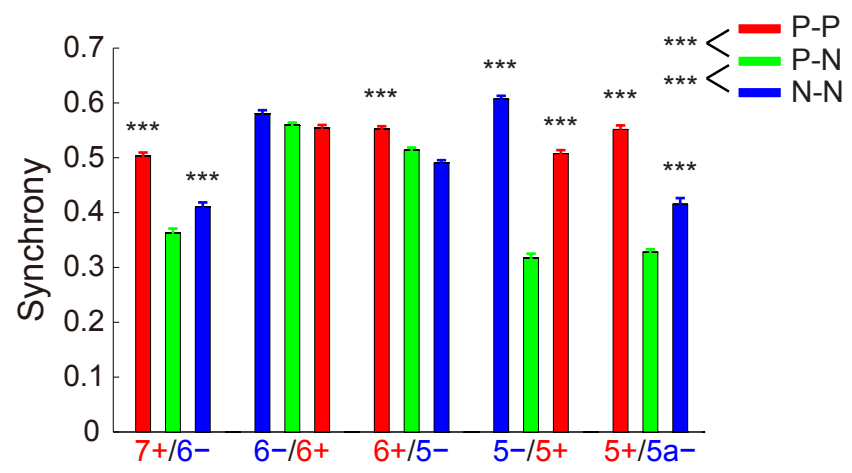

$6+/ 5-$

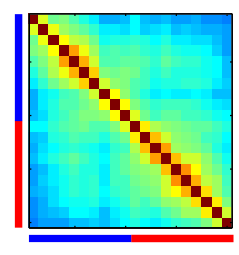

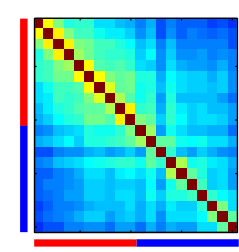

C
$5-/ 5+$

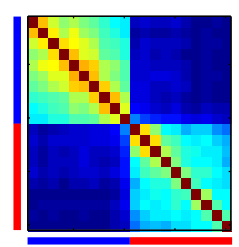

$5+/ 5 a-$

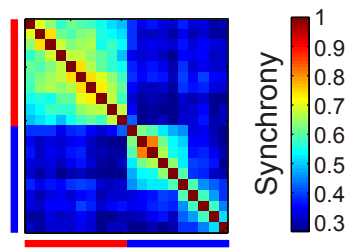

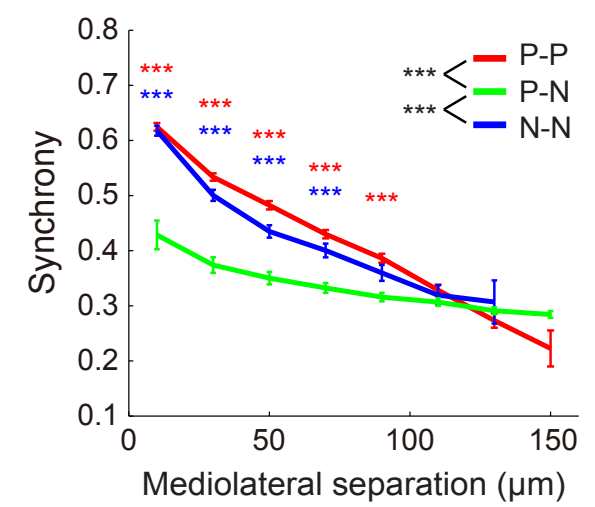

D

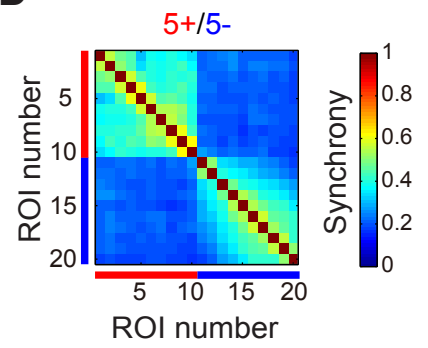

E

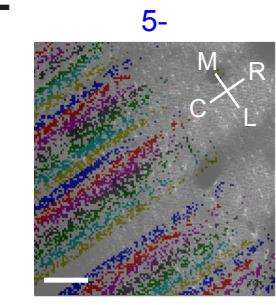

$5-$

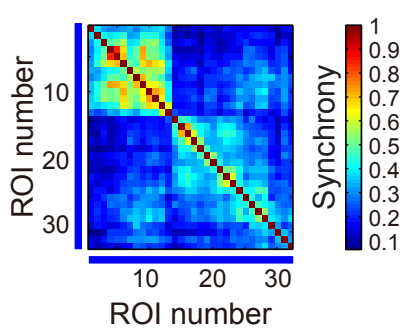

Figure 3. Relationships between aldolase C compartments and complex spike synchrony. $A$, Correlation matrices between $\Delta F / F$ waves from 20 adjacent dendrites across aldolase $C$ boundaries; 10 dendrites each from both sides of the boundaries in Crus Ila of the left hemisphere. Data are averaged across mice. Red and blue bars represent aldolase C-positive and -negative PCs, respectively; $n=6,7,8,6$, and 3 mice for the $7+/ 6-, 6-16+, 6+/ 5-, 5-15+$, and $5+/ 5 a-$ boundaries, respectively. $B$, Synchrony between $\Delta F / F$ waves from $P C$ dendrite pairs whose mediolateral distance were $<100 \mu \mathrm{m}$ and located within and across the aldolase ( compartments. Data are represented as mean \pm SEM. P-P (red bars), synchrony of $\Delta F / F$ waves between the aldolase C-positive PCs; P-N (green bars), that across aldolase C-positive and -negative PCs; and N-N (blue bars), that between the aldolase C-negative PCs. ${ }^{* * *} p<0.001$, two-way ANOVA followed by post hoc Tukey's sest. C, Calcium transient synchrony plotted against the mediolateral separation between PC dendrite pairs. Data from $7+/ 6-, 5-15+$, and $5+/ 5 \mathrm{a}-$ boundaries are averaged ( 4670 dendrite pairs, $n=12$ mice). P-P (red trace), P-N (green trace), and N-N (blue trace) represent synchrony between aldolase C-positive to -positive, aldolase C-positive to -negative, and aldolase C-negative to -negative PC dendrite pairs, respectively. Data are averaged within $20 \mu \mathrm{m}$ bin and represented as mean \pm SEM. Red and blue asterisks denote significant difference between P-P and P-N, and between N-N and P-N, respectively. ${ }^{* * *} p<0.001$, two-way ANOVA followed by posthoc Tukey's test. $D$, Same as $A$, but only $5+/ 5-$ boundary in Crus lla of right hemisphere; $n=6$ mice. $\boldsymbol{E}$, Left, Calcium imaging using Cal-520 was performed within a single compartment (5-) in Crus lla of right hemisphere. Pseudocolored ROls are overlaid to the corresponding RFP image. Right, Correlation matrix calculated by using $\Delta F / F$ waves from all the dendrite pairs in the corresponding images on the left. Blue bars represent aldolase C-negative PCs. R, rostral; $C$, caudal; L, lateral; and M, medial. Scale bar, $40 \mu \mathrm{m}$.

in the mediolateral direction was significantly higher within the aldolase $\mathrm{C}$ compartments than across the compartments for overall dataset (Fig. 3B). Post hoc comparison showed the significant difference in $7+/ 6-, 5-/ 5+$, and $5+/ 5 a-$ boundaries. This was true for any group of dendritic pairs located with distances $<80 \mu \mathrm{m}$ in these representative areas (Fig. $3 C$ ). However, synchrony between distant $(>100 \mu \mathrm{m})$ PC pairs within the aldolase $\mathrm{C}$ compartments was comparable to those across the aldolase $\mathrm{C}$ boundaries (Fig. $3 \mathrm{C}$ ). The result may reflect the fact that each aldolase $\mathrm{C}$ compartment with $300-$ $400 \mu \mathrm{m}$ in width contains multiple microzones of $\sim 100 \mu \mathrm{m}$ in width. In line with this notion, we found more than one highsynchrony area with clear boundary within single aldolase $\mathrm{C}$ compartments (5a-; Fig. 3A, right, 5-; Fig. 3E, right).
Neither carbenoxolone nor harmaline influences the structure-function relationship

Next, we asked why spontaneous complex spike synchrony is finely delineated by some of the aldolase $\mathrm{C}$ boundaries. A previous study suggests that electrical coupling between inferior olivary neurons is responsible for the synchronous complex spike firing between closely located PCs (Ozden et al., 2009). We therefore examined the effect of a gap-junction blocker, carbenoxolone, on complex spike synchrony. Previous studies show that carbenoxolone blocks gap junctions in the inferior olive and reduces coupling between inferior olivary neurons (Blenkinsop and Lang, 2006; Hashizume et al., 2013). However, we did not observe any change in the frequency of dendritic calcium transient (Fig. 4A) and the boundary of synchrony in relation to that 
A

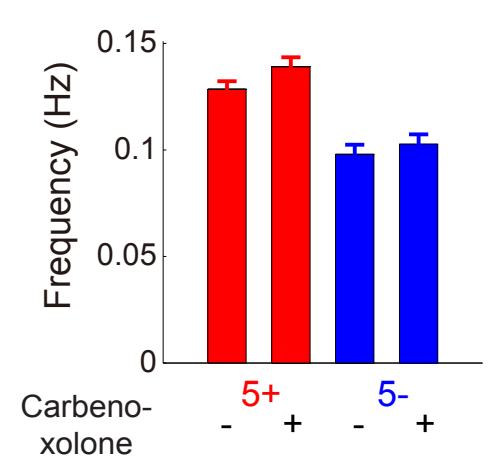

C

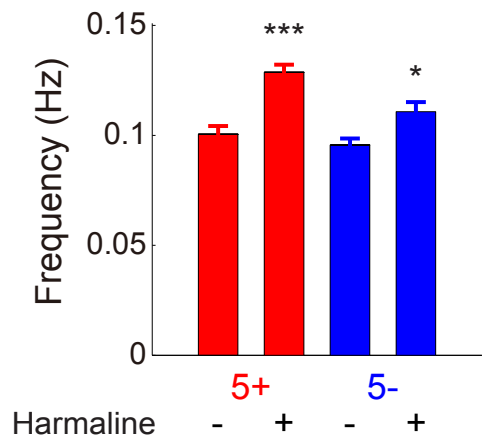

B

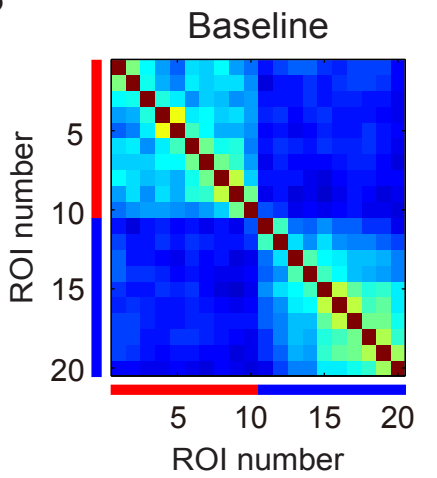

D

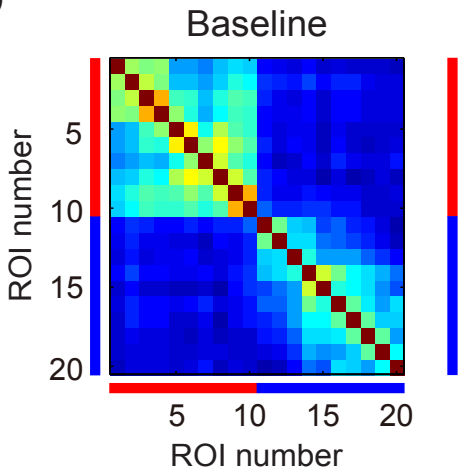

Carbenoxolone

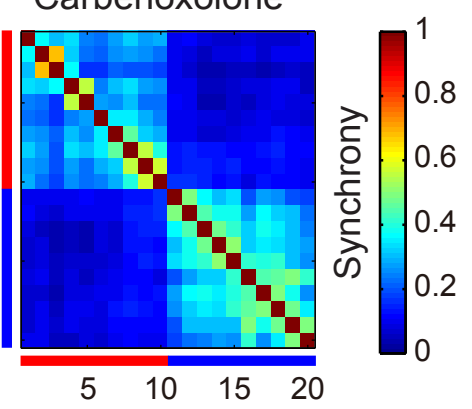

Harmaline

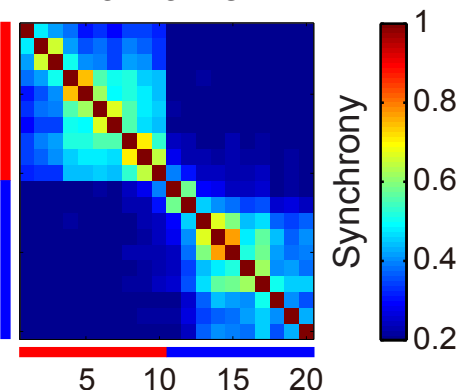

Figure 4. Relationship between ald olase ( compartments and complex spike synchrony is unchanged after application of carbenoxolone or harmaline. $A$, Averaged frequency of calcium transient in PCs within aldolase (-positive (red) and -negative (blue) compartments before and after carbenoxolone application (baseline: 49 and 44 cells, carbenoxolone: 52 and 41 cells for $5+$ and $5-$ compartment, respectively; $n=3$ mice). Data are represented as mean \pm SEM. No significant difference was observed (Mann-Whitney $U$ test). $\boldsymbol{B}$, Correlation matrices averaged across mice ( $n=$ 3 mice) before and after carbenoxolone application. Red and blue bars denote aldolase C-positive and -negative PCs, respectively. $\boldsymbol{C}$, Same as $\boldsymbol{A}$, but for harmaline (baseline: 46 and 38 cells; harmaline: 50 and 43 cells for $5+$ and $5-$ compartment, respectively; $n=3$ mice). Data are represented as mean \pm SEM $,{ }^{*} p<0.05,{ }^{* * *} p<0.001$, Mann-Whitney $U$ test. $\boldsymbol{D}$, Same as $\boldsymbol{B}$, but for harmaline ( $n=3$ mice). Note that the boundaries of synchrony are unaffected by injections of either carbenoxolone or harmaline.

of aldolase C compartment after carbenoxolone injection (Fig. $4 B$ ). Other possible mechanisms for the synchronous complex spike firing are common inputs to weakly coupled inferior olivary neurons or finely delineated climbing fiber projection from inferior olivary subnucleus to PCs. We then examined the effect of harmaline that enhances rhythm-generating ionic current of inferior olivary neurons (Miwa, 2007) and thus increases inputindependent firing of these neurons. We found that firing rate of dendritic calcium transient was significantly increased in both the aldolase C-positive and -negative compartments after harmaline application (Fig. 4C). However, the boundary of complex spike synchrony remained unchanged (Fig. 4D). These results suggest that the boundary of complex spike synchrony at that of aldolase $\mathrm{C}$ compartments is largely maintained by finely delineated projection pattern of climbing fibers from individual inferior olivary subnuclei to the corresponding aldolase $\mathrm{C}$ compartments.

\section{Sensory-evoked responses and microzones at aldolase C compartment boundaries}

The cerebellar folium Crus IIa of rodents processes tactile sensory information from the perioral region (Brown and Bower, 2001). This folium is therefore a suitable location to investigate the relationship between aldolase $\mathrm{C}$ compartments and microzones activated by sensory stimulation. For this purpose, we stimulated the ipsilateral perioral surface using air puffs while performing calcium imaging at aldolase $\mathrm{C}$ compartment boundaries (Fig. $5 A, B)$. Sensory-evoked calcium transients were exclusively ob- served at a particular cluster of dendrites corresponding to classical zones/microzones (Andersson and Oscarsson, 1978; Fig. $5 C)$. Notably, sensory-evoked calcium transients were observed most consistently in several PCs in the 6- compartment (Fig. $5 D)$. Furthermore, the response profile was sharply delineated at the $7+/ 6-$ boundary (Fig. $5 D$, left).

We next calculated the synchrony of calcium transients during sensory stimulation and compared it to that of spontaneous events. A subtracted correlation matrix was generated and it clearly shows that synchrony of calcium transients within the 6compartment at the $7+/ 6-$ boundary was specifically increased during sensory stimulation, representing a sensory-evoked microzone (Fig. 5E). In this area, the boundaries of sensory-evoked microzones precisely corresponded to those of the aldolase $\mathrm{C}$ compartments. The synchrony of sensory-evoked calcium transients also tended to be higher than that of spontaneous events at other boundaries but overall microzonal structures remained unchanged (Fig. 5E).

\section{Coactivation of microzones across aldolase $\mathrm{C}$ compartments in awake mice}

We examined if sensory response was dependent on a vigilance state. Mice were acclimated to head fixation before experiments and temporarily awoken after imaging under anesthesia when they received air-puff stimuli. During awake state, the sensory response rate was increased and response profile was extended beyond the aldolase $\mathrm{C}$ boundary (Fig. 6A). We next examined whether microzonal organization was also modulated during 
A

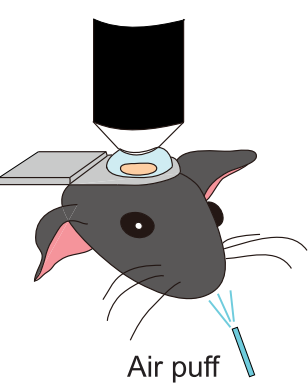

stimulation
B

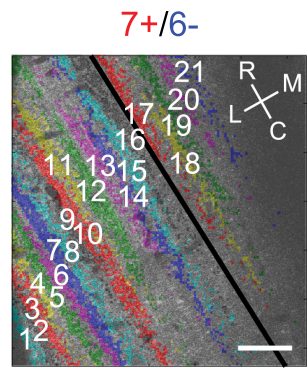

D

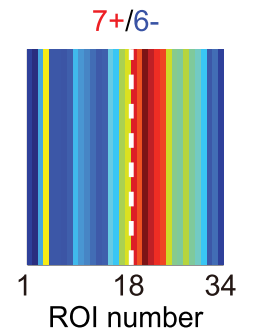

E
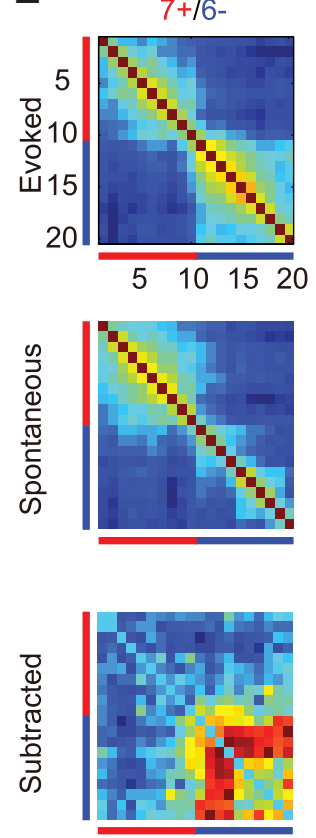

$6-16+$

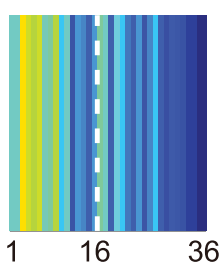

6-/6+
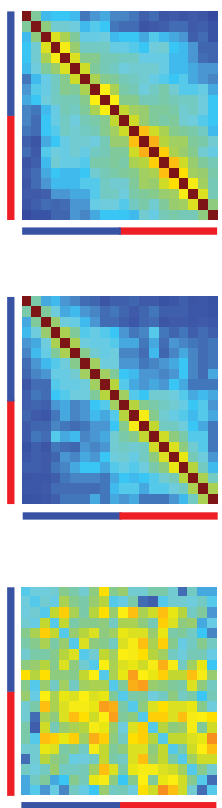

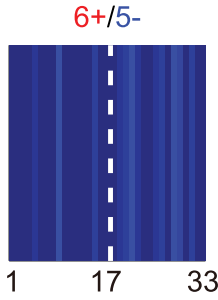

$6+/ 5-$
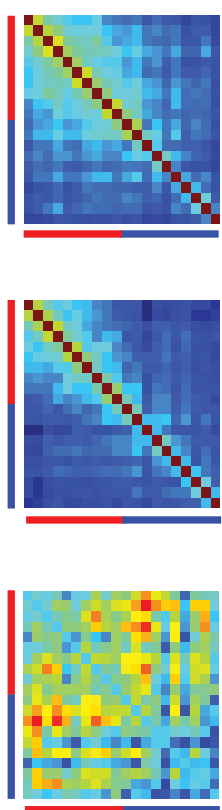

C

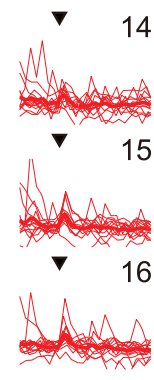

5-/5+

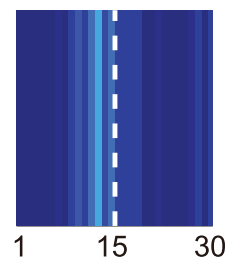

$5-/ 5+$
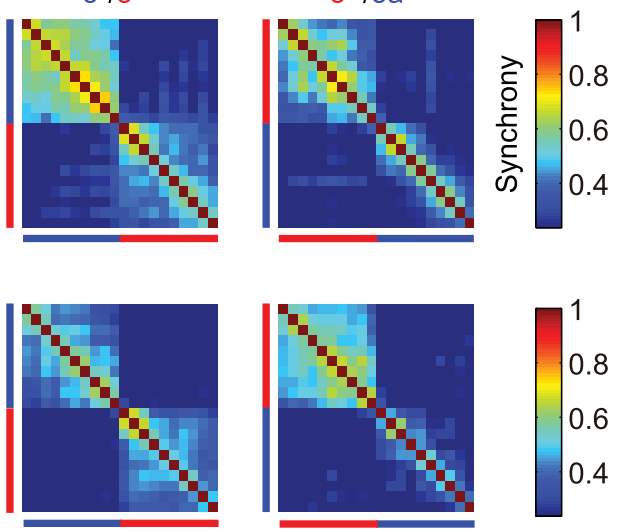

$5+/ 5 a-$
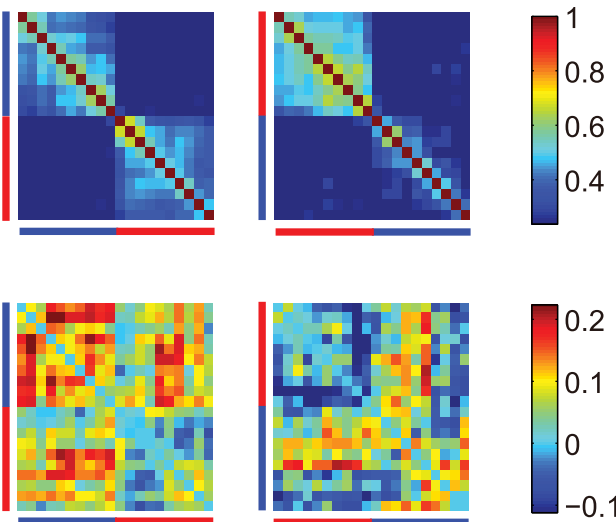
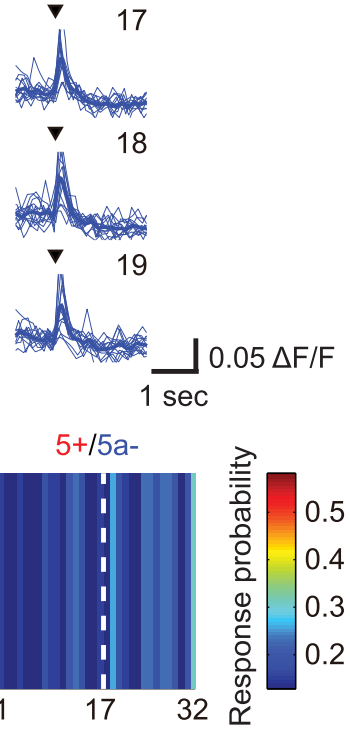
. 
A $7+16$

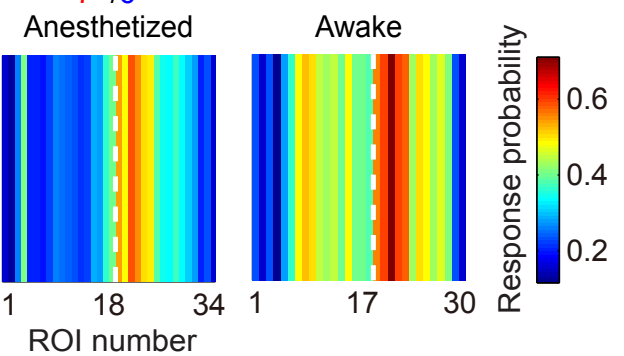

B

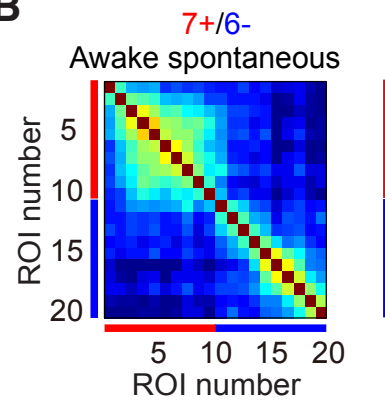

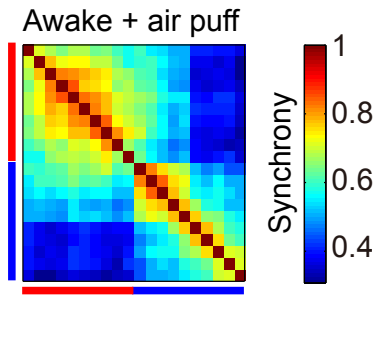

C
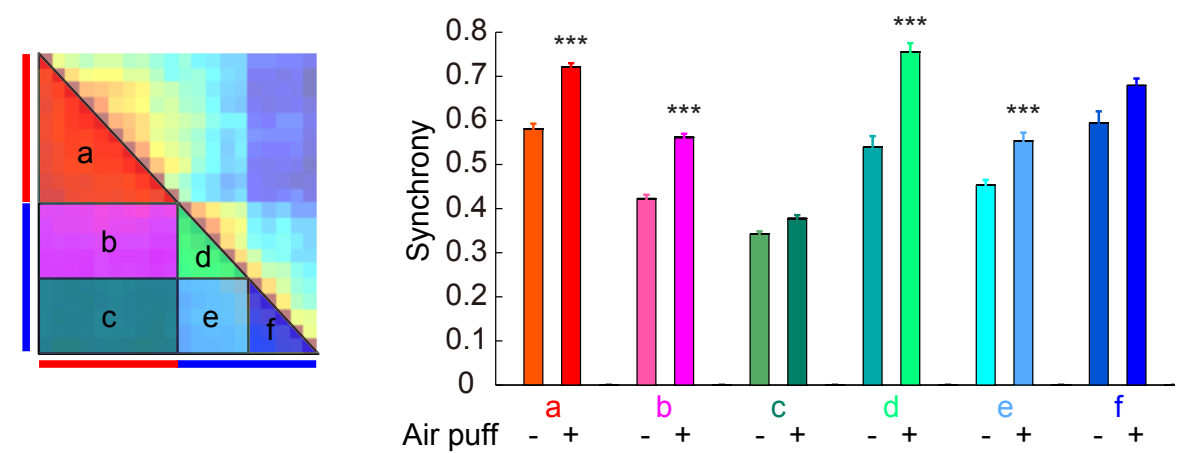

Figure 6. Microzones are coactivated during sensory stimulation in awake mice. $\boldsymbol{A}$, Left, Color-coded response probability of PCs around the $7+/ 6-$ aldolase $C$ boundary in Crus $I I$ (same data as Fig. 5D, left). Right, Same as the left, but during awake state. White broken lines represent the aldolase (boundary; $n=6$ and 3 mice for anesthetized and awake states, respectively. $\boldsymbol{B}$, Correlation matrices of calcium transients in PCs at the $7+16$ - boundary in awake mice during periods with (right) and without (left) air-puff stimulation. Red and blue bars represent dendrites in aldolase (-positive and -negative compartments, respectively; $n=3$ mice. $\boldsymbol{C}$, Right, Averaged complex spike synchrony calculated using matrices in $\boldsymbol{B}$ within areas depicted on the left ( $\boldsymbol{a}-\boldsymbol{f}$ ), without $(-)$ and with (+) air-puff stimulation. Data are represented as mean $\pm \mathrm{SEM}$; ${ }^{* *} p<0.001$, two-way ANOVA followed by post hoc Tukey's test.

could be coactivated during sensory stimulation in awake animals.

\section{Visualization of cerebellar zones in live mice}

Although the function of aldolase C/zebrin II in the cerebellum is still largely unclear, other physiologically important molecules (e.g., mGluR1b, phospholipase $\mathrm{C} \beta 3 / 4$, protein kinase $\mathrm{C} \delta$, and glutamate transporter EAAT4) in PCs are known to exhibit the same or complementary stripe-shaped expression patterns as aldolase C (Chen and Hillman, 1993; Sarna et al., 2006; Paukert et al., 2010; Wang et al., 2011). Thus aldolase C compartmentalization is of the same functional importance as above molecules in the cerebellar cortex. Indeed, a recent study has shown that firing properties of PCs depend on the expression of aldolase C (Zhou et al., 2014). These previous studies required post hoc anatomical identification of aldolase $\mathrm{C}$ compartments in perfused brain sections. In contrast, our Aldoc-tdTomato mice have great advantages for the functional analysis of cerebellar cortex, because they enable us to identify aldolase C-positive and -negative PCs in vivo and to make targeted imaging or recordings from these cells in an intact cerebellum. Furthermore, since we used a "red" fluorescent protein, tdTomato, to visualize aldolase C-positive cells, "green" functional probes such as GCaMP can be easily used in the same mice. The use of aldolase $\mathrm{C}$ as a marker for cerebellar functional modules also facilitates manipulation (e.g., optogenetics) of cerebellar functions related to motor coordination and learning (Tsubota et al., 2013).

\footnotetext{
Aldolase $\mathrm{C}$ compartments represent physiological zones in the cerebellar cortex

We found that the boundaries of aldolase $\mathrm{C}$ compartments corresponded at single-cell resolution to those of complex spike syn-
}

chrony (Figs. 2E, 3A, 3D). Our results from the experiments with carbenoxolone and harmaline suggest that the boundaries of complex spike synchrony in reference to the aldolase C compartments are mainly attributed to finely delineated climbing fiber projection (Fig. 4). We also found the aldolase $\mathrm{C}$ boundaries, which did not show clear correspondence to complex spike synchrony (Fig. 3;6-/6+ and 6+/5-). Some dendrites of inferior olivary neurons, where gap junctions are formed, are known to extend beyond the subnucleus (Sugihara et al., 2007). This may explain why complex spikes are synchronized across the boundaries of these aldolase $\mathrm{C}$ compartments.

Synchronous complex spike firing in large numbers of PCs results in rebound firing of deep cerebellar nuclear neurons, which is thought to be crucial for cerebellar-dependent learning (Bengtsson et al., 2011). Facilitated coupling within subnuclei of the inferior olive may provide a basis for modular activation of cerebellar cortex, which has significant implications for cortical information processing. In the present study, we confirmed that sensory responses and sensory-evoked microzones were activated completely in line with the aldolase C compartments (Fig. 5). Microzones are narrower than the aldolase C compartments ( 100 vs $300-$ $400 \mu \mathrm{m})$. Synchrony between distant $(>100 \mu \mathrm{m})$ PC dendrites was almost the same within and across the aldolase $\mathrm{C}$ compartments (Fig. 3C). Moreover, we found more than one high-synchrony area within a single aldolase $\mathrm{C}$ compartment (Fig. 3E). We propose that aldolase $\mathrm{C}$ compartments represent zones consisting of several functional microzones, which may represent submodalities.

\section{Microzones can be reorganized and coordinated during awake state}

We found that the neighboring microzones were consistently coactivated across aldolase $\mathrm{C}$ boundaries during sensory stimu- 
lation in awake mice (Fig. $6 B$ ). The sensory response profile also extended beyond the aldolase $\mathrm{C}$ boundary (Fig. $6 \mathrm{~A}$ ), which may reflect a higher probability of complex spike generation in PCs or an increase in sensory-evoked climbing fiber inputs during awake state. However, increases in complex spike synchrony during sensory stimulation in awake state showed more restricted pattern compared with the overall increase in sensory-evoked complex spike responses in PCs around the $7+/ 6-$ boundary (Fig. $6 B, C)$. Thus, degree of complex spike synchrony itself may also be modulated by sensory stimulus during the awake state. Extensive projections are known to exist from motor and association areas of the cerebral cortex to the lateral cerebellum (Sasaki et al., 1975), the region we examined in the present study. It is therefore possible that electrotonic coupling between neuronal ensembles in the inferior olive are modulated by inputs from the cerebral cortex depending on vigilance or behavioral state of the animal. Presumed weak coupling between tightly coupled cell assemblies might be enhanced, leading to the elevation of synchrony between these assemblies (De Zeeuw et al., 2011). Coactivation of neighboring microzones implies the facilitation of coupling between microzones with different (e.g., sensory and motor) modalities under awake conditions. This flexible coordination of microzones may further facilitate cerebellum-dependent sensorimotor processing and learning. Application of highly efficient motor tasks (Isomura et al., 2009; Hira et al., 2013) will further elucidate the relationship between coordinated microzonal activities and sensorimotor functions.

\section{References}

Andersson G, Oscarsson O (1978) Climbing fiber microzones in cerebellar vermis and their projection to different groups of cells in the lateral vestibular nucleus. Exp Brain Res 32:565-579. CrossRef Medline

Bell CC, Kawasaki T (1972) Relations among climbing fiber responses of nearby Purkinje cells. J Neurophysiol 35:155-169. Medline

Bengtsson F, Ekerot CF, Jörntell H (2011) In vivo analysis of inhibitory synaptic inputs and rebounds in deep cerebellar nuclear neurons. PLoS One 6:e18822. CrossRef Medline

Blenkinsop TA, Lang EJ (2006) Block of inferior olive gap junctional coupling decreases Purkinje cell complex spike synchrony and rhythmicity. J Neurosci 26:1739-1748. CrossRef Medline

Brochu G, Maler L, Hawkes R (1990) Zebrin II: a polypeptide antigen expressed selectively by Purkinje cells reveals compartments in rat and fish cerebellum. J Comp Neurol 291:538-552. CrossRef Medline

Brown IE, Bower JM (2001) Congruence of mossy fiber and climbing fiber tactile projections in the lateral hemispheres of the rat cerebellum. J Comp Neurol 429:59-70. CrossRef Medline

Chen S, Hillman DE (1993) Compartmentation of the cerebellar cortex by protein kinase C $\delta$. Neuroscience 56:177-188. CrossRef Medline

de Zeeuw CI, Holstege JC, Ruigrok TJ, Voogd J (1989) Ultrastructural study of the GABAergic, cerebellar, and mesodiencephalic innervation of the cat medial accessory olive: anterograde tracing combined with immunocytochemistry. J Comp Neurol 284:12-35. CrossRef Medline

De Zeeuw CI, Hoebeek FE, Bosman LW, Schonewille M, Witter L, Koekkoek SK (2011) Spatiotemporal firing patterns in the cerebellum. Nat Rev Neurosci 12:327-344. CrossRef Medline

Fujita H, Aoki H, Ajioka I, Yamazaki M, Abe M, Oh-Nishi A, Sakimura K, Sugihara I (2014) Detailed expression pattern of aldolase C (Aldoc) in the cerebellum, retina and other areas of the CNS studied in Aldoc-Venus knock-in mice. PLoS One 9:e86679. CrossRef Medline

Fukaya M, Tsujita M, Yamazaki M, Kushiya E, Abe M, Akashi K, Natsume R, Kano M, Kamiya H, Watanabe M, Sakimura K (2006) Abundant distribution of TARP $\gamma 8$ in synaptic and extrasynaptic surface of hippocampal neurons and its major role in AMPA receptor expression on spines and dendrites. Eur J Neurosci 24:2177-2190. CrossRef Medline

Ghosh KK, Burns LD, Cocker ED, Nimmerjahn A, Ziv Y, Gamal AE, Schnitzer MJ (2011) Miniaturized integration of a fluorescence microscope. Nat Methods 8:871-878. CrossRef Medline

Groenewegen HJ, Voogd J, Freedman SL (1979) The parasagittal zonation within the olivocerebellar projection. II. Climbing fiber distribution in the intermediate and hemispheric parts of cat cerebellum. J Comp Neurol 183:551-601. CrossRef Medline

Hashimoto M, Mikoshiba K (2003) Mediolateral compartmentalization of the cerebellum is determined on the "birth date" of Purkinje cells. J Neurosci 23:11342-11351. Medline

Hashizume M, Miyazaki T, Sakimura K, Watanabe M, Kitamura K, Kano M (2013) Disruption of cerebellar microzonal organization in GluD2 (GluR82) knockout mouse. Front Neural Circuits 7:130. CrossRef Medline

Hira R, Ohkubo F, Ozawa K, Isomura Y, Kitamura K, Kano M, Kasai H, Matsuzaki M (2013) Spatiotemporal dynamics of functional clusters of neurons in the mouse motor cortex during a voluntary movement. J Neurosci 33:1377-1390. CrossRef Medline

Isomura Y, Harukuni R, Takekawa T, Aizawa H, Fukai T (2009) Microcircuitry coordination of cortical motor information in self-initiation of voluntary movements. Nat Neurosci 12:1586-1593. CrossRef Medline

Kitamura K, Häusser M (2011) Dendritic calcium signaling triggered by spontaneous and sensory-evoked climbing fiber input to cerebellar Purkinje cells in vivo. J Neurosci 31:10847-10858. CrossRef Medline

Kitamura K, Judkewitz B, Kano M, Denk W, Häusser M (2008) Targeted patch-clamp recordings and single-cell electroporation of unlabeled neurons in vivo. Nat Methods 5:61-67. CrossRef Medline

Mishina M, Sakimura K (2007) Conditional gene targeting on the pure C57BL/6 genetic background. Neurosci Res 58:105-112. CrossRef Medline

Miwa H (2007) Rodent models of tremor. Cerebellum 6:66-72. CrossRef Medline

Mukamel EA, Nimmerjahn A, Schnitzer MJ (2009) Automated analysis of cellular signals from large-scale calcium imaging data. Neuron 63:747760. CrossRef Medline

Oberdick J, Baader SL, Schilling K (1998) From zebra stripes to postal zones: deciphering patterns of gene expression in the cerebellum. Trends Neurosci 21:383-390. CrossRef Medline

Ozden I, Lee HM, Sullivan MR, Wang SS (2008) Identification and clustering of event patterns from in vivo multiphoton optical recordings of neuronal ensembles. J Neurophysiol 100:495-503. CrossRef Medline

Ozden I, Sullivan MR, Lee HM, Wang SS (2009) Reliable coding emerges from coactivation of climbing fibers in microbands of cerebellar Purkinje neurons. J Neurosci 29:10463-10473. CrossRef Medline

Paukert M, Huang YH, Tanaka K, Rothstein JD, Bergles DE (2010) Zones of enhanced glutamate release from climbing fibers in the mammalian cerebellum. J Neurosci 30:7290-7299. CrossRef Medline

Pologruto TA, Sabatini BL, Svoboda K (2003) ScanImage: flexible software for operating laser scanning microscopes. Biomed Eng Online 2:13. CrossRef Medline

Sarna JR, Marzban H, Watanabe M, Hawkes R (2006) Complementary stripes of phospholipase $C \beta 3$ and $C \beta 4$ expression by Purkinje cell subsets in the mouse cerebellum. J Comp Neurol 496:303-313. CrossRef Medline

Sasaki K, Oka H, Matsuda Y, Shimono T, Mizuno N (1975) Electrophysiological studies of the projections from the parietal association area to the cerebellar cortex. Exp Brain Res 23:91-102. Medline

Sasaki K, Bower JM, Llinás R (1989) Multiple Purkinje Cell Recording in Rodent Cerebellar Cortex. Eur J Neurosci 1:572-586. CrossRef Medline

Schultz SR, Kitamura K, Post-Uiterweer A, Krupic J, Häusser M (2009) Spatial pattern coding of sensory information by climbing fiber-evoked calcium signals in networks of neighboring cerebellar Purkinje cells. J Neurosci 29:8005-8015. CrossRef Medline

Smith SL, Häusser M (2010) Parallel processing of visual space by neighboring neurons in mouse visual cortex. Nat Neurosci 13:1144-1149. CrossRef Medline

Sugihara I (2011) Compartmentalization of the deep cerebellar nuclei based on afferent projections and aldolase C expression. Cerebellum 10:449463. CrossRef Medline

Sugihara I, Quy PN (2007) Identification of aldolase C compartments in the mouse cerebellar cortex by olivocerebellar labeling. J Comp Neurol 500: 1076-1092. CrossRef Medline

Sugihara I, Shinoda Y (2004) Molecular, topographic, and functional organization of the cerebellar cortex: a study with combined aldolase $\mathrm{C}$ and olivocerebellar labeling. J Neurosci 24:8771-8785. CrossRef Medline 
Sugihara I, Wu HS, Shinoda Y (2001) The entire trajectories of single olivocerebellar axons in the cerebellar cortex and their contribution to cerebellar compartmentalization. J Neurosci 21:7715-7723. Medline

Sugihara I, Marshall SP, Lang EJ (2007) Relationship of complex spike synchrony bands and climbing fiber projection determined by reference to aldolase C compartments in crus IIa of the rat cerebellar cortex. J Comp Neurol 501:13-29. CrossRef Medline

Tada M, Takeuchi A, Hashizume M, Kitamura K, Kano M (2014) A highly sensitive fluorescent indicator dye for calcium imaging of neural activity in vitro and in vivo. Eur J Neurosci 39:1720-1728. CrossRef Medline

Tsubota T, Ohashi Y, Tamura K (2013) Optogenetics in the cerebellum: Purkinje cell-specific approaches for understanding local cerebellar functions. Behav Brain Res 255:26-34. CrossRef Medline
Voogd J, Glickstein M (1998) The anatomy of the cerebellum. Trends Neurosci 21:370-375. CrossRef Medline

Voogd J, Pardoe J, Ruigrok TJ, Apps R (2003) The distribution of climbing and mossy fiber collateral branches from the copula pyramidis and the paramedian lobule: congruence of climbing fiber cortical zones and the pattern of zebrin banding within the rat cerebellum. J Neurosci 23:46454656. Medline

Wang X, Chen G, Gao W, Ebner TJ (2011) Parasagittally aligned, mGluR1dependent patches are evoked at long latencies by parallel fiber stimulation in the mouse cerebellar cortex in vivo. J Neurophysiol 105:1732-1746. CrossRef Medline

Zhou H, Lin Z, Voges K, Ju C, Gao Z, Bosman LW, Ruigrok TJ, Hoebeek FE, De Zeeuw CI, Schonewille M (2014) Cerebellar modules operate at different frequencies. Elife:e02536. CrossRef 